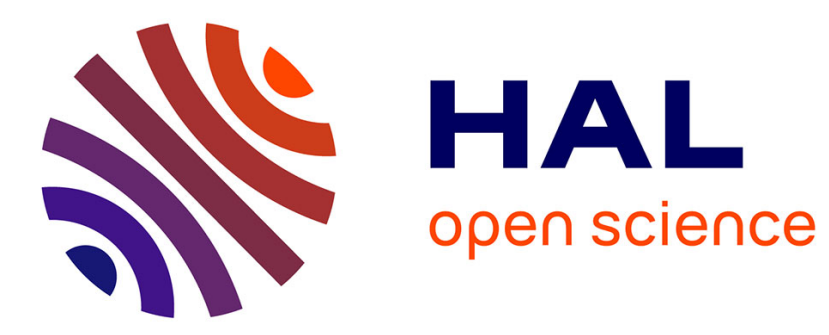

\title{
Technique de mesure des faibles conductibilités électriques sous haute pression
}

\author{
G. Mesnard, E. Souquet, G. Noyel
}

\section{To cite this version:}

G. Mesnard, E. Souquet, G. Noyel. Technique de mesure des faibles conductibilités électriques sous haute pression. Revue de Physique Appliquée, 1971, 6 (3), pp.365-368. 10.1051/rphysap:0197100603036500 . jpa-00243557

\section{HAL Id: jpa-00243557 https://hal.science/jpa-00243557}

Submitted on 1 Jan 1971

HAL is a multi-disciplinary open access archive for the deposit and dissemination of scientific research documents, whether they are published or not. The documents may come from teaching and research institutions in France or abroad, or from public or private research centers.
L'archive ouverte pluridisciplinaire HAL, est destinée au dépôt et à la diffusion de documents scientifiques de niveau recherche, publiés ou non, émanant des établissements d'enseignement et de recherche français ou étrangers, des laboratoires publics ou privés. 


\title{
TECHNIQUE DE MESURE DES FAIBLES CONDUCTIBILITÉS ÉLECTRIQUES SOUS HAUTE PRESSION
}

\author{
G. MESNARD, E. SOUQUET et G. NOYEL \\ Université de Lyon I, Laboratoire de Physique Electronique
}

(Reçu le 26 mars 1971)

\begin{abstract}
Résumé. - On propose des solutions à des problèmes importants qui se posent dans l'étude des propriétés électriques de corps très peu conducteurs avec un appareillage de mesure sous pression hydrostatique : réalisation de sorties électriques bien isolées, choix du fluide transmetteur de pression, cellule de mesures pour liquides.

Abstract. - We give solutions of important problems appearing in the study of electrical properties of low conductivity materials with an apparatus for measurements under hydrostatic pressure : preparation of electrical leads with a good insulation, choice of the fluid transmitting the pressure, cell for measurements on liquids.
\end{abstract}

Afin de procéder à des mesures de conductibilité électrique sur des solides moléculaires (pérylène, anthracène, ...) et des liquides (carbures aromatiques et aliphatiques, alcools, ...) présentant des résistances souvent supérieures à $10^{13} \Omega$, nous avons été amenés à étudier :

1) des sorties électriques possédant un très bon isolement électrique,

2) des fluides transmetteurs de pression hydrostatique présentant une haute résistivité électrique, ainsi qu'une grande inertie chimique,

3) une technologie particulière pour l'étude des liquides, permettant d'une part de travailler dans des conditions de grande propreté et d'autre part d'utiliser une faible quantité de liquide.

I. Sorties électriques. - Le premier système que nous avons essayé, proposé par Cugan [1], fait appel à des isolants tels que l'araldite qui, liquide dans son état non polymérisé, donne ainsi une plus grande facilité de montage. Les résultats sont décevants, car on n'a pu dépasser une pression de 5 kbars, tout particulièrement aux températures supérieures à $50^{\circ} \mathrm{C}$. Les isolements électriques sont rarement supérieurs à $10^{13} \Omega$. D'autres isolants, tels que le Rhodester 1111 ou l'araldite $\mathrm{AT}_{1}$, ont été essayés, mais, si la résistance mécanique est meilleure, l'isolement obtenu n'a jamais donné satisfaction, la géométrie des montages étant difficile à respecter.

Nous avons donc abandonné ce principe pour reprendre celui du cône métallique isolé, déjà proposé par plusieurs auteurs [2], [3], [4], [5] et d'emploi assez généralisé. Différents isolants ont été essayés, notamment le téflon, abandonné rapidement à cause

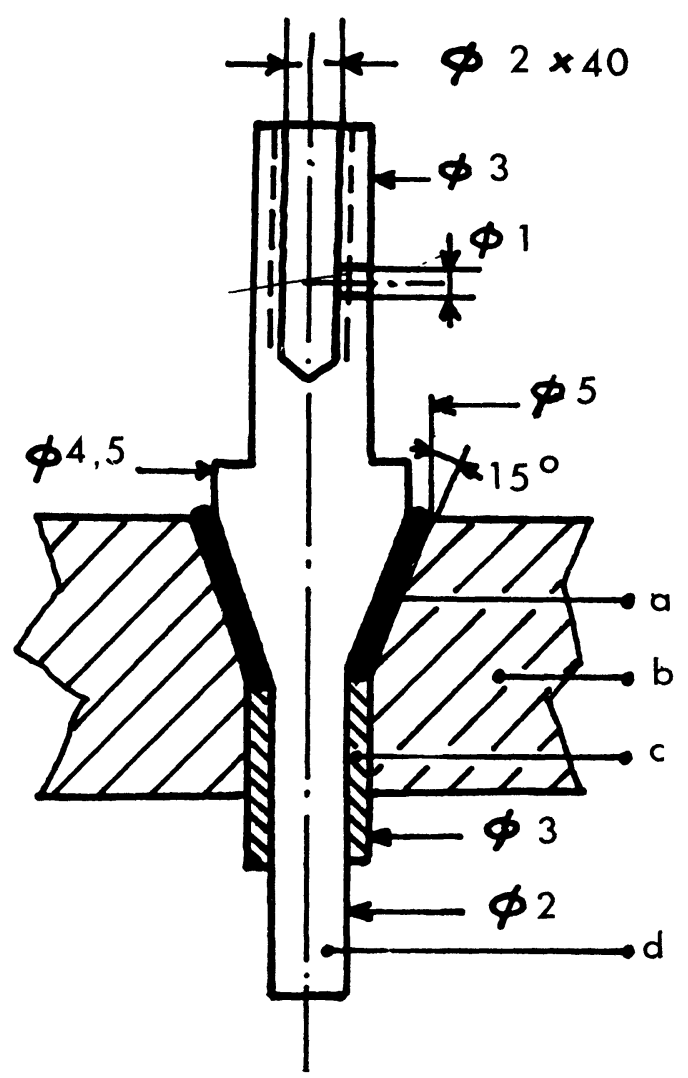

FIG. 1. - Sorties électriques isolées. $a$ : araldite AT $1 ; b$ : culasse ; $c$ : gaine téflon; $d$ : électrode en acier.

d'un fluage excessif, et l'araldite liquide, dont la tenue en température laisse particulièrement à désirer. Le Rhodester 1111 a de remarquables qualités mécaniques, tout particulièrement lorsqu'il est chargé avec de l'alumine ou du quartz finement divisés; 
il peut être employé couramment à $150^{\circ} \mathrm{C}$ sans que ses qualités mécaniques soient fortement altérées. Mais c'est une résine relativement hygroscopique, ce qui ne nous a pas permis de dépasser des isolements de $10^{11}$ à $10^{12} \Omega$.

A l'heure actuelle nous utilisons l'Araldite AT1 en poudre, pure ou mélangée à $30 \%$ de quartz en poudre, ce qui permet d'atteindre sans difficulté une pression de 12 kbars et une température de $140^{\circ} \mathrm{C}$. L'épaisseur d'isolant est de l'ordre de 0,3 $\mathrm{mm}$. La pente des cônes a été fixée à une valeur importante $\left(15^{\circ}\right)$, ce qui permet d'éviter l'extrusion de l'isolant.

Le croquis coté 1 montre clairement le montage. Avec les dimensions indiquées, on place facilement 7 électrodes de sortie dans une enceinte de $20 \mathrm{~mm}$ de diamètre.

La technologie de montage est simple. On place les électrodes dans leur logement, sans les enfoncer complètement, on introduit l'araldite dans l'espace laissé libre (Fig. $2 a$ et $2 b$ ), puis l'ensemble est porté

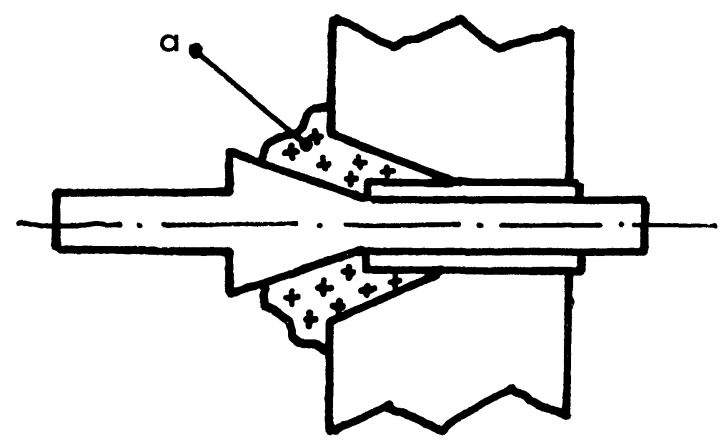

FIG. 2a. - Araldite en poudre.

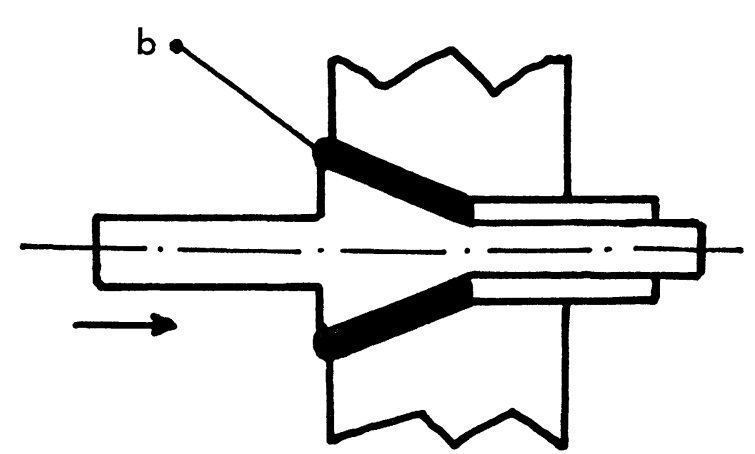

FIG. 2b. - Araldite polymérisée.

à une température de $80^{\circ} \mathrm{C}$, à laquelle l'araldite devient liquide. Les électrodes sont alors enfoncées jusqu'à leur position définitive. La température est ensuite portée à $140^{\circ}$ pendant $12 \mathrm{~h}$; l'araldite est alors polymérisée. Un nettoyage aux ultra-sons dans un bain d'acétone permet d'enlever les couches d'oxydation superficielles. L'opération finale consiste à vieillir les électrodes sous une pression et à une température supérieures à celles qui sont prévues par la suite.

II. Fluides transmetteurs de pression. - Nous avons envisagé différents liquides qui doivent posséder les qualités suivantes :

a) forte résistivité électrique,

b) grande inertie chimique,

c) bonne tenue en température,

d) viscosité acceptable à des pressions élevées.

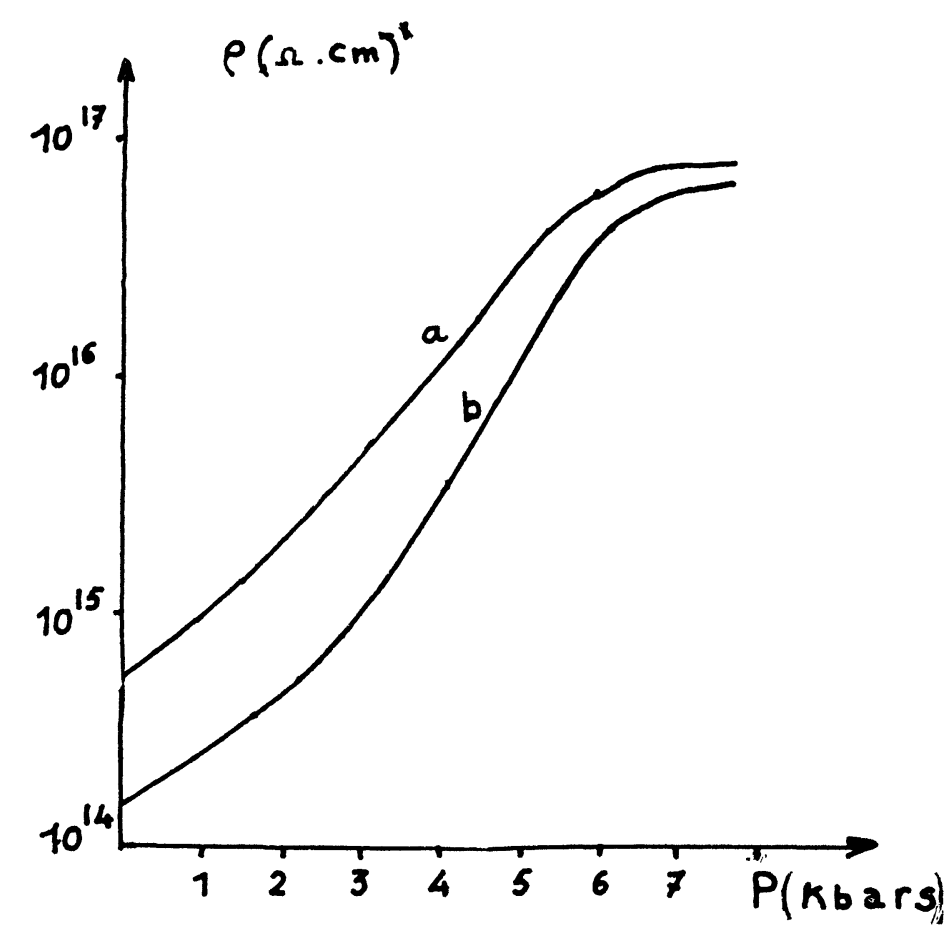

Fig. 3. - Caractéristique $\log \rho=\mathrm{f}(P)$ du fluide SI 200, $20 \mathrm{csk}$, pour les températures de $20^{\circ}(a)$ et $40^{\circ}(b)$. 
A ce titre, ont été essayés, le pentane, l'isopentane, des alcools possédant une ramification comme l'alcool isopropylique, puis des fluides silicones SI 510 et SI 200 de viscosités différentes. Finalement nous avons retenu le fluide SI 200 utilisé à 20 csk, car pour une viscosité de 0,5 csk il présente un léger pouvoir dissolvant sur certains des corps étudiés (carbures aromatiques), au-delà de $50^{\circ} \mathrm{C}$.

La figure 3 donne la caractéristique de résistivité $\rho$ en fonction de la pression $P$ pour deux températures $\left(20^{\circ} \mathrm{C}\right.$ et $\left.40^{\circ} \mathrm{C}\right)$. Jusqu'à 6 kbars, c'est sensiblement une droite puis la courbe s'infléchit. La partie supérieure quasi horizontale paraît correspondre à une forte viscosité. Ceci se comprend bien si on suppose que la conductibilité est essentiellement ionique.

Bridgmann [6] et d'autres ont montré que l'on obtient une courbe semblable pour le logarithme de la viscosité ; la résistivité varie donc comme la viscosité.

III. Mesures de résistivité sur les liquides. - Différents impératifs sont à respecter pour les mesures de résistivités sur des échantillons liquides :

a) maintenir un très grand état de pureté pour l'échantillon,

b) éliminer tout effet parasite, en particulier les courants de fuite, ainsi que la dissolution des électrodes,

c) n'employer que de faibles quantités du liquide.

Tous ces impératifs ont pu être respectés par l'emploi d'une cellule spéciale d'essais (Fig. 4). Cette cellule, remplie du liquide à étudier, est placée dans l'enceinte entourée par le fluide transmetteur de pression. L'étanchéité totale de cette cellule permet d'éviter toute souillure due au fluide environnant et à l'arrachement du métal lors de la descente du piston.

La géométrie particulière de la construction et le dimensionnement permettent de limiter les courants de fuite; on a pu éviter l'emploi d'un anneau de garde. L'espace compris entre les électrodes cylindriques est de $1 \mathrm{~mm}$ avec des diamètres respectifs de 7 et $9 \mathrm{~mm}$; la hauteur de la surface active est de $10 \mathrm{~mm}$. D'autre part, pour les liquides très résistants, on prendra la précaution de gainer en téflon les électrodes de sortie, afin de rendre négligeables les fuites électriques dues au fluide transmetteur de pression. L'utilisation de quartz et de colles " epoxy » comme support de cellule permet d'améliorer encore les caractéristiques.

D'autre part les électrodes sont en or pur massif ; les premiers essais, effectués avec des électrodes en cuivre doré, ont montré que, même avec des couches d'or de plusieurs microns d'épaisseur, le cuivre pouvait se trouver au contact de la solution.

Le capuchon supérieur de la cellule est usiné dans du téflon, la calotte supérieure ayant une épaisseur très faible $(0,2$ à $0,3 \mathrm{~mm})$, afin qu'elle puisse se déformer facilement et transmettre la pression dans de bonnes conditions au liquide placé dans la cellule.

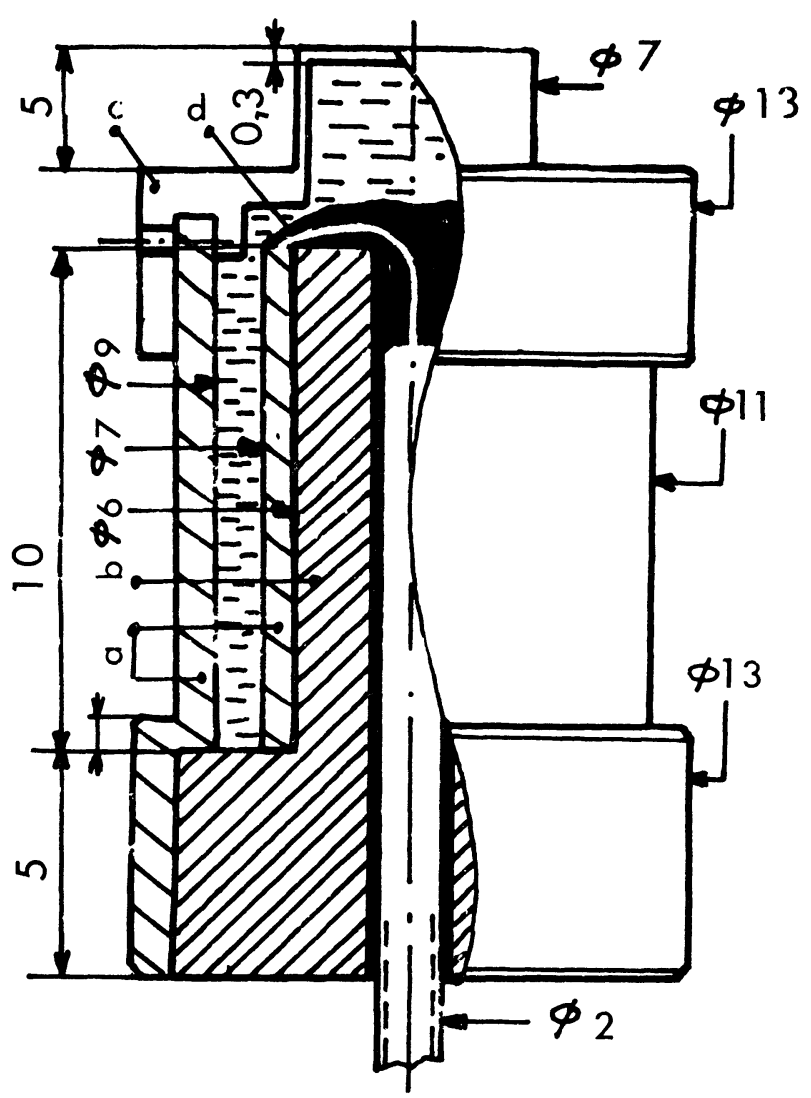

Fig. 4. - Cellule d'étude de la conductibilité électrique des liquides. $a$ : or ; $b:$ quartz; $c$ : téflon; $d$ : colle epoxy.

Le remplissage de la cellule s'effectue à l'aide d'une seringue, par le trou ménagé dans le flanc de la calotte en téflon, celle-ci étant préalablement relevée. Elle est ensuite enfoncée; l'excédent de liquide est éjecté par le trou de remplissage. On a pris soin de garder une bonne interférence de diamètre entre l'anneau d'or et le capuchon de téflon. Celui-ci se dilatant beaucoup sous l'effet de la température à la pression atmosphérique, on risque une perte d'étanchéité. On peut facilement pallier à cet inconvénient en prévoyant un ressort de serrage.

Les cotes peuvent être facilement connues à $0,01 \mathrm{~mm}$ près, ce qui laisse présumer d'une bonne précision quant aux mesures de résistance $R$ effectuées. La résistivité du liquide inclus est donnée par la formule :

$$
\rho=\frac{2 \pi l R}{\ln \frac{r_{2}}{r_{1}}},
$$

où $r_{1}$ et $r_{2}$ sont les 2 rayons des cylindres et $l$ leur longueur. 


\section{Bibliographie}

[1] Cugan (C. D.), J. Sci. Instr., 1956, 33, 1606.

[2] Davis (L. A.), Gordon (R. B.), TiEen (J. K.), VidNYs (J. R.), Rev. Sc. Instr., 1964, 35, 368.

[3] Welbergen (H. J.), J. Sci. Instr., 1933, 10, 247.

[4] CoRnish (R. A.), Ruoff (A. L.), Rev. Sci. Instr., 1961, 32, 639 .
[5] Scott (G. J.), BARB (S. E.), Rev. Sci. Instr., 1961, 32, 868.

[6] Bridgmann (P. W.), The Physics of high Pressure, G. Bell and Sons, London, 1952. 